\title{
Condições de trabalho e saúde dos agentes de saúde: Uma revisão integrativa
}

\author{
Work and health conditions of health care agents: An integrative review \\ Condiciones laborales y agentes sanitarios: Una revisión integradora
}

Fernanda Queiroz Rego de Sousa Lopes

ORCID: https://orcid.org/0000-0003-3432-0291 Universidade Estadual de Feira de Santana, Brasil E-mail: fernandaqor@yahoo.com.br

Tânia Maria de Araújo

ORCID: https://orcid.org/0000-0003-2766-7799 Universidade Estadual de Feira de Santana, Brasil E-mail: araujo.tania@uefs.br

Sheila Santa Barbara Cerqueira ORCID: https://orcid.org/0000-0002-3557-7200 Universidade Estadual de Feira de Santana, Brasil E-mail: sheilinhabio@yahoo.com.br

Elaine Andrade Leal Silva

ORCID: https://orcid.org/0000-0003-2551-1264 Universidade Federal do Recôncavo da Bahia, Brasil E-mail: elainesilva@hotmail.com

Paloma de Sousa Pinho Freitas

ORCID: https://orcid.org/0000-0001-6402-0869 Universidade Federal do Recôncavo da Bahia, Brasil E-mail: paloma@ufrb.edu.br

Margarete Costa Heliotério

ORCID: https://orcid.org/0000-0001-6102-4346 Universidade Federal do Recôncavo da Bahia, Brasil E-mail: mcssantos@ufrb.edu.br

\begin{abstract}
Resumo
Objetivo: Analisar a produção científica nacional e internacional referente às condições de trabalho e saúde dos agentes de saúde, no período de 2007 a 2017. Metodologia: Revisão integrativa das condições de trabalho e saúde dos agentes de saúde, por meio de busca eletrônica de artigos indexados nas bases de dados PUBMED, MEDLINE, SCIELO e LILACS, utilizando os descritores primary health care, occupational health, health personnel, community health workers, em inglês, no período de outubro a dezembro de 2017. Após aplicação dos critérios de inclusão, exclusão e leitura na integra 26 artigos fizeram parte desta revisão, e foram analisados conforme formulário padronizado, com dados referentes à autoria, periódico, ano de publicação, população, objetivo e metodologia. As informações extraídas foram submetidas a análise de conteúdo categorial. Resultados: Observou-se predominância de estudos publicados no Brasil, no estado de São Paulo, de abordagem quantitativa. Desse material, 18 estudos trataram das condições de saúde e 15 apontaram fatores de risco no trabalho. Dentre as condições inadequadas destacaram as dificuldades impostas por morar e trabalhar no território, situações ambientais, exposição à radiação solar, violência e riscos ergonômicos. Os transtornos mentais relacionados ao trabalho entre os agentes de saúde foram os problemas de saúde mais estudados, seguidos dos problemas de pele, queixas de voz e das lesões do sistema osteomuscular. Conclusões: Verificou-se que as condições de trabalho e saúde dos agentes de saúde apontam para a necessidade de mudanças nos processos de trabalho, a fim de minimizar potenciais efeitos negativos sobre a saúde, especialmente a saúde mental.

Palavras-chave: Saúde do trabalhador; Trabalhador da saúde; Agentes comunitários de saúde; Condições de trabalho; Atenção primária à saúde.
\end{abstract}

\footnotetext{
Abstract

Objective: To analyze national and international scientific production regarding the working and health conditions of health agents, from 2007 to 2017. Methodology: Integrative review of the working and health conditions of health agents, through an electronic search for articles indexed in the PUBMED, MEDLINE, SCIELO and LILACS databases, using the descriptors primary health care, occupational health, health personnel, community health workers, in english, from october to december 2017. After applying the inclusion and exclusion criteria and full reading 26 articles were part of this review, and were analyzed according to a standardized form, with data referring to authorship, journal, year of publication, population, objective and methodology. The extracted information was submitted to categorical content analysis. Results: There was a predominance of studies published in Brazil, in the state of São Paulo, with a quantitative approach. Of this material, 18 studies addressed health conditions and 15 pointed to risk factors at work. Among the
} 
inadequate conditions, they highlighted the difficulties imposed by living and working in the territory, environmental situations, exposure to solar radiation, violence and ergonomic risks. Work-related mental disorders among health workers were the most studied health problems, followed by skin problems, voice complaints and musculoskeletal system injuries. Conclusions: It was found that the working conditions and health of health agents point to the need for changes in work processes, in order to minimize potential negative effects on health, especially mental health.

Keywords: Occupational health; Health worker; Community health agents; Work conditions; Primary health care.

\section{Resumen}

Objetivo: Analizar la producción científica nacional e internacional sobre las condiciones de trabajo y salud de los agentes de salud, de 2007 a 2017. Metodología: Revisión integradora de las condiciones de trabajo y salud de los agentes de salud, a través de una búsqueda electrónica de artículos indexados en PUBMED, MEDLINE, bases de datos SCIELO y LILACS, utilizando los descriptores primary health care, occupational health, health personnel, community health workers, en inglés, de octubre a diciembre de 2017. Después de aplicar los criterios de inclusión y exclusión y lectura completa 26 artículos fueron parte de esta revisión, y fueron analizados según formulario estandarizado, con datos referentes a autoría, revista, año de publicación, población, objetivo y metodología. La información extraída fue sometida al análisis de contenido categórico. Resultados: Predominaron los estudios publicados en Brasil, en el estado de São Paulo, con abordaje cuantitativo. De ese material, 18 estudios abordaron condiciones de salud y 15 señalaron factores de riesgo en el trabajo. Entre las condiciones inadecuadas destacaron las dificultades impuestas por vivir y trabajar en el territorio, situaciones ambientales, exposición a la radiación solar, violencia y riesgos ergonómicos. Los trastornos mentales relacionados con el trabajo entre los trabajadores de la salud fueron los problemas de salud más estudiados, seguidos de los problemas de la piel, las quejas de la voz y las lesiones del sistema músculo esquelético. Conclusiones: Se constató que las condiciones de trabajo y de salud de los agentes de salud apuntan a la necesidad de cambios en los procesos de trabajo, con el fin de minimizar potenciales efectos negativos sobre la salud, especialmente la salud mental.

Palabras clave: Salud ocupacional; Trabajador de la salud; Agentes comunitarios de salud; Condiciones de trabajo; Primeros auxilios.

\section{Introdução}

O agente de saúde (AS) é um trabalhador dos serviços de saúde, que desempenha papel de protagonista do cuidado, tanto no nível coletivo, quanto individual, ao desenvolver ações de apoio à saúde das comunidades mais vulneráveis. Fazem parte dos AS os agentes comunitários de saúde (ACS) e os agentes de combate a endemias (ACE). Esses trabalhadores realizam atividades peculiares na prestação do cuidado junto às equipes de saúde da família, pois atuam diretamente em contato com as famílias, em seus domicílios, vivenciando o mesmo contexto social, por residirem no território em que desenvolvem suas atividades laborais (Santos, 2015).

O processo de trabalho dos AS mudou de espaços fechados e internos para os espaços abertos e externos as unidades de saúde a partir da implementação do Sistema Único de Saúde (SUS), com a criação do programa de agentes comunitários de saúde (PACS), programa de saúde da família (PSF) e da estratégia de saúde da família (ESF), com a atuação dos ACS mais próximos à população, uma vez que um dos pré-requisitos para a ocupação destes cargos é residir e trabalhar no mesmo território (Santos, 2015).

No Brasil, os AS são fundamentais na consolidação dos princípios do SUS, desenvolvendo o trabalho centrado nas visitas domiciliares, com o papel de mediar a comunicação entre a comunidade e os serviços de saúde, tendo como atribuições: cadastrar famílias, registrar informações, prestar orientações, agendar consultas, promover ações de vigilância à saúde, de vigilância ambiental e controle de endemias. No cenário internacional, também são mediadores entre comunidade e serviços de saúde, distribuem medicamentos e acompanham as famílias nos tratamentos (Samudio et al., 2017).

Para que o trabalho dos AS seja feito de acordo com o preconizado pelo Ministério da Saúde, é necessário que as condições sob as quais ele é executado sejam adequadas. Em condições de trabalho inadequadas, nas quais se observa um desequilíbrio entre as exigências da atividade laboral e as cargas de trabalho físicas, químicas, biológicas, mecânicas, fisiológicas e psíquicas no qual o trabalho é executado, desencadeia-se o desgaste do trabalhador com perda da sua capacidade biopsíquica e social, podendo desencadear processos de adoecimento laboral (Facchini, 2005). 
O trabalho na saúde dos AS tem sido apontado como fonte de adoecimento devido a condições de trabalho em ambientes insalubres expostos às intempéries climáticas, fatores da organização do trabalho envolvendo agressões físicas, verbais e problemas interpessoais (Assunção, 2016; Samudio et al., 2017). Tais condições predispõem aos transtornos mentais relacionados ao trabalho, doenças do sistema osteomuscular, problemas de pele e aos acidentes de trabalho (Assunção, 2016).

Os AS foram a categoria alvo deste estudo, pela importância que exercem no SUS, tanto no Brasil, como em outros países que tem sistemas de saúde fortemente baseados na Atenção Primária à Saúde (APS) ${ }^{1}$. No Brasil os ACS e os ACE são os AS que compõem as equipes de saúde da atenção primária e da vigilância a saúde (Assunção, 2016).

Diante do exposto é fundamental dar visibilidade às condições de trabalho que os AS vivenciam e aos processos de adoecimento relacionado ao trabalho, para que se possa investir em medidas para a proteção e cuidado destinados a esta categoria profissional. Além do mais, as transformações recentes na organização do trabalho em saúde e as incorporações de novas tecnologias de cuidado podem implicar no surgimento de novos problemas de saúde, o que torna imperativa a necessidade de novos estudos e revisões bibliográficas. Nesse sentido, este estudo tem como objetivo analisar a produção científica nacional e internacional referente às condições de trabalho e saúde dos AS no período de 2007 a 2017.

\section{Metodologia}

Revisão integrativa da literatura com foco na saúde dos trabalhadores e trabalhadoras AS. A revisão integrativa permite sintetizar o que já foi produzido sobre determinado tema, oferecendo informações abrangentes, com descrição do estado da arte em que os autores com maior expertise no assunto apontam como mais relevante, com embasamento científico, para estruturar e conduzir tomada de decisões, além de evidenciar lacunas no conhecimento (Botelho; Cunha; Macedo, 2011; Roman; Mendes et al., 2008).

Os procedimentos metodológicos desenvolveram-se em seis etapas: (1) identificação do tema e seleção da questão de pesquisa; (2) definição dos critérios de inclusão e exclusão; (3) identificação dos estudos pré-selecionados e selecionados; (4) categorização dos estudos selecionados; (5) análise e interpretação dos resultados; e (6) apresentação da revisão/síntese do conhecimento (Mendes et al. 2008; Botelho, Cunha, Macedo, 2011).

A primeira etapa dessa revisão consistiu em definir uma pergunta clara, elaborada com conhecimento prévio, visando orientar as estratégias de busca e seleção dos artigos (Macedo; 2011). Baseado nisto, elaborou-se a seguinte questão norteadora para este estudo: $O$ que se conhece sobre as condições de saúde e trabalho dos/as trabalhadores/as agentes de saúde?

Os descritores utilizados na pesquisa estão disponíveis nas bases de dados informatizadas dos Descritores em Ciências da Saúde (DeCs), da Biblioteca Virtual em Saúde (BVS). Para busca dos artigos foram utilizados os descritores em combinações por meio do marcador boleano "AND”. Utilizou-se os descritores: Primary Health Care, Occupational Health, Health Personnel e Community Health Workers, todas na língua inglesa e os termos limitados por aspas, a fim de realizar uma busca com o termo na íntegra.

Foram selecionados estudos que atendessem aos seguintes critérios de inclusão: artigos nacionais e internacionais, publicados no período de 2007 a 2017, disponibilizados na íntegra, em inglês e/ou português, incluindo estudos qualitativos e/ou quantitativos. Foram excluídos artigos repetidos, teses, dissertações, anais de congresso, resenhas e revisões de literatura. As bases de dados utilizados para esta revisão integrativa foram Medical Literature Analysis and Retrievel System Online (MEDLINE/Pubmed), Scientific Electronic Library Online (SCIELO) e Centro Latino-Americano de Informação em Saúde

\footnotetext{
1. APS corresponde aos cuidados essenciais à saúde, baseados em tecnologias acessíveis, que levam os serviços de saúde o mais próximo possível dos lugares de vida e trabalho das pessoas, constituindo, assim, o primeiro nível de contato com o sistema nacional de saúde e o primeiro elemento de um processo contínuo de atenção (Alma-Ata, 1978).
} 
(LILACS). Os dados foram coletados no período de outubro a dezembro de 2017. Iniciou-se a estratégia de busca com os critérios predefinidos. O primeiro resultado identificou 1.610 publicações.

Após a leitura sistemática dos títulos, foram excluídos os artigos repetidos, permanecendo um total de 247 publicações. Depois da exclusão pela leitura dos resumos, foram selecionados 62 artigos para serem lidos na íntegra. Nesta última etapa, foram mantidos na revisão 26 artigos. Desta forma, realizaram-se todas as etapas necessárias (da pré-seleção a leitura dos textos na íntegra), buscando-se selecionar aqueles que contemplavam a temática e a pergunta norteadora pré-definida (Botelho, Cunha, Macedo, 2011).

$\mathrm{Na}$ análise do material identificado, foram elaborados quadros-síntese, com as seguintes características: título, autoria, periódico de publicação, população, com número de indivíduos que participaram do estudo, metodologia, objetivos e país de realização do estudo. Também foi elaborado um quadro contendo as categorias analíticas investigadas, com a finalidade de responder as questões norteadoras. Realizou-se a análise e interpretação dos dados referentes aos estudos selecionados, por meio da análise de conteúdo (Bardin, 2012).

As categorias analíticas definidas foram: condições de trabalho dos AS e o adoecer dos AS. Na categoria "condições de trabalho" buscou-se na literatura publicações que convergiam sobre como se dá o processo de trabalho dos AS, considerando as exposições aos fatores de riscos físicos, químicos, biológicos, ergonômicos, acidentes e os psicossociais. Com relação a categoria "o adoecer dos agentes de saúde" buscou-se sumarizar, com base na literatura, as doenças relacionadas ao trabalho que acometem esta categoria profissional.

Quanto aos preceitos éticos, realizou-se a coleta utilizando dados secundários e públicos nas bases de dados online. Como se tratam de dados públicos que não identificam ou comprometem a integridade de qualquer pessoa e nem trazem prejuízos para as instituições e autores (as) das publicações utilizadas, não foi necessário submeter ao Comitê de Ética em Pesquisa.

\section{Resultados}

$\mathrm{Na}$ revisão integrativa foram selecionados para análise 26 artigos que atenderam aos objetivos do estudo. No que se refere a origem geográfica 22 estudos foram publicações brasileiras e apenas (4) quatro estudos foram publicações internacion ais. Entre os estudos publicados no Brasil, a maior frequência foi no estado de São Paulo (36,4\%), seguido do Rio de Janeiro (22,7\%), Minas Gerais $(13,6 \%)$ e Rio Grande do Sul $(13,6 \%)$. Quanto ao tipo de abordagem, os estudos quantitativos predominaram $(73,0 \%)$. Dentre os veículos de publicações, o destaque foi para a Revista de Enfermagem da Universidade Estadual do Rio de Janeiro e Cadernos de Saúde Pública.

Do material obtido na revisão integrativa, considerando as características dos trabalhadores, dois estudos abordaram trabalhadores comunitários de saúde, um, na China e outro, no Canadá. Outro estudo realizado no Irã pesquisou os Behvarzes (funcionários locais do Irã, familiarizados com as necessidades de saúde rural), e um estudo na Gambia foi feito com oficiais de saúde ambiental. Entre os estudos brasileiros, 19 artigos focalizaram ACS, um ACE e dois estudos ambos os grupos de trabalhadores.

Com relação à temática, 18 estudos trataram das condições de saúde dos AS, e 15 abordaram os fatores de risco para os problemas de saúde observados (Quadro 1). 
Quadro 1. Estudos sobre a saúde de agentes de saúde segundo autor (es) e ano, título, população e objetivo, identificados na revisão integrativa, 2017.

\begin{tabular}{|c|c|c|c|c|c|}
\hline ARTIGO & $\begin{array}{c}\text { AUTOR(ES) } \\
\text { /ANO }\end{array}$ & TITULO & POPULAÇÃO/ N & PAIS & OBJETIVO \\
\hline A1 & $\begin{array}{l}\text { Trindade et al., } \\
2007\end{array}$ & $\begin{array}{l}\text { Cargas de trabalho entre os } \\
\text { agentes comunitários de } \\
\text { saúde }\end{array}$ & $\begin{array}{c}\text { ACS } \\
05\end{array}$ & Brasil & $\begin{array}{l}\text { Identificar as cargas de trabalho a que } \\
\text { estão submetidos e promover ações que } \\
\text { os despertassem para o autocuidado. }\end{array}$ \\
\hline A2 & $\begin{array}{l}\text { Nascimento; } \\
\text { David, } 2008\end{array}$ & $\begin{array}{l}\text { Avaliação de riscos no } \\
\text { trabalho dos agentes } \\
\text { comunitários de Saúde: um } \\
\text { processo participativo }\end{array}$ & $\begin{array}{c}\text { ACS } \\
26\end{array}$ & Brasil & $\begin{array}{l}\text { Descrever e analisar o processo } \\
\text { metodológico de desenvolvimento de um } \\
\text { instrumento para avaliação de riscos no } \\
\text { trabalho dos agentes comunitários de } \\
\text { saúde. }\end{array}$ \\
\hline A3 & $\begin{array}{l}\text { Silva; Menezes, } \\
2008\end{array}$ & $\begin{array}{l}\text { Burnout syndrome and } \\
\text { common mental disorders } \\
\text { among community-based } \\
\text { health agents. }\end{array}$ & $\begin{array}{c}\text { ACS } \\
141\end{array}$ & Brasil & $\begin{array}{l}\text { Estimar a prevalência da síndrome do } \\
\text { esgotamento profissional e de transtornos } \\
\text { mentais comuns em ACS, e fatores } \\
\text { associados. }\end{array}$ \\
\hline A4 & $\begin{array}{c}\text { Lancman et al., } \\
2009\end{array}$ & $\begin{array}{l}\text { Repercussões da violência } \\
\text { na saúde mental de } \\
\text { trabalhadores do Programa } \\
\text { Saúde da Família }\end{array}$ & $\begin{array}{c}\text { ACS } \\
97\end{array}$ & Brasil & $\begin{array}{l}\text { Descrever formas de violência externa e } \\
\text { indireta que afetam a saúde mental de } \\
\text { trabalhadores do Programa de Saúde da } \\
\text { Família, bem como as estratégias } \\
\text { desenvolvidas pelos trabalhadores para } \\
\text { viabilizar seu trabalho e se proteger } \\
\text { psicologicamente. }\end{array}$ \\
\hline A5 & $\begin{array}{c}\text { Telles; Pimenta, } \\
2009\end{array}$ & $\begin{array}{l}\text { Síndrome de Burnout em } \\
\text { agentes comunitários } \\
\text { saúde e estratégias de } \\
\text { enfrentamento }\end{array}$ & $\begin{array}{c}\text { ACS } \\
80\end{array}$ & Brasil & $\begin{array}{l}\text { Descrever a ocorrência da Síndrome de } \\
\text { Burnout ACS e suas estratégias de } \\
\text { enfrentamento. }\end{array}$ \\
\hline A6 & $\begin{array}{c}\text { Wai; Carvalho, } \\
2009\end{array}$ & $\begin{array}{l}\text { O trabalho do agente } \\
\text { comunitário de saúde: } \\
\text { fatores de sobrecarga e } \\
\text { estratégias } \\
\text { enfrentamento }\end{array}$ & $\begin{array}{c}\text { ACS } \\
17\end{array}$ & Brasil & $\begin{array}{l}\text { Identificar, por meio das percepções dos } \\
\text { ACS, eventos que provocam sobrecarga e } \\
\text { as formas de lidar com eles. }\end{array}$ \\
\hline A7 & Lima et al., 2010 & $\begin{array}{l}\text { Fotoexposição solar e } \\
\text { fotoproteção de agentes de } \\
\text { saúde em município de } \\
\text { Minas Gerais }\end{array}$ & $\begin{array}{c}\text { ACS e ACE } \\
13\end{array}$ & Brasil & $\begin{array}{l}\text { Conhecer os hábitos de exposição solar de } \\
\text { ACS e ACE, que atuam no município de } \\
\text { Ribeirão das Neves, Minas Gerais. }\end{array}$ \\
\hline A8 & Drebitet al., 2010 & $\begin{array}{l}\text { Fatores de risco } \\
\text { ocupacionais e ambientais } \\
\text { para quedas entre os } \\
\text { trabalhadores do setor de } \\
\text { saúde }\end{array}$ & $\begin{array}{l}\text { Trabalhador } \\
\text { comunitário da } \\
\text { saúde } \\
411\end{array}$ & Canadá & $\begin{array}{l}\text { Identificar os aspectos ocupacionais e } \\
\text { ambientais relacionados a quedas. }\end{array}$ \\
\hline A9 & $\begin{array}{l}\text { Malakoutet al., } \\
2011\end{array}$ & $\begin{array}{l}\text { Job stress and burnout } \\
\text { syndrome in a sample of } \\
\text { rural health workers, } \\
\text { behvarzes, in Tehran, Iran. }\end{array}$ & $\begin{array}{l}\text { Trabalhadores de } \\
\text { saúde rural } \\
227\end{array}$ & Irã & $\begin{array}{l}\text { Determinar o nível de burnout, estado de } \\
\text { saúde mental e o estresse ocupacional } \\
\text { entre trabalhadores de saúde rural. }\end{array}$ \\
\hline A10 & Ge et al., 2011 & $\begin{array}{l}\text { Fatores associados à } \\
\text { satisfação no trabalho entre } \\
\text { os trabalhadores da saúde } \\
\text { da comunidade chinesa: um } \\
\text { estudo transversal. }\end{array}$ & $\begin{array}{l}\text { Trabalhador } \\
\text { comunitário de } \\
\text { saúde } \\
2.100\end{array}$ & China & $\begin{array}{l}\text { Descrever o nível de satisfação no } \\
\text { trabalho e seus fatores associados entre } \\
\text { trabalhadores da saúde da comunidade } \\
\text { chinesa numa região metropolitana } \\
\text { (Shenyang) e uma pequena cidade } \\
\text { (Benxi) na província de Liaoning. }\end{array}$ \\
\hline A11 & $\begin{array}{l}\text { Santos; David, } \\
2011\end{array}$ & $\begin{array}{l}\text { Percepções do estresse no } \\
\text { trabalho dos agentes } \\
\text { comunitários de saúde }\end{array}$ & $\begin{array}{c}\text { ACS } \\
32\end{array}$ & Brasil & $\begin{array}{l}\text { Identificar os fatores de estresse } \\
\text { ocupacional referidos por ACS e analisar } \\
\text { a sua relação com possíveis efeitos na } \\
\text { saúde, segundo sua percepção. }\end{array}$ \\
\hline A12 & $\begin{array}{l}\text { Cipriano; Ferreira, } \\
2011 .\end{array}$ & $\begin{array}{l}\text { Queixas de voz em agentes } \\
\text { comunitários de saúde: } \\
\text { correlação entre problemas } \\
\text { gerais de saúde, hábitos de } \\
\text { vida e aspectos vocais }\end{array}$ & $\begin{array}{c}\text { ACS } \\
28\end{array}$ & Brasil & $\begin{array}{l}\text { Conhecer as queixas de voz e } \\
\text { correlacioná-las à autor-referência de } \\
\text { problemas gerais de saúde, hábitos de } \\
\text { vida e aspectos vocais, num grupo de } \\
\text { ACS do município de São Paulo. }\end{array}$ \\
\hline A13 & Lopes et al., 2012 & $\begin{array}{l}\text { Agentes Comunitários de } \\
\text { Saúde e as vivências de } \\
\text { prazer - sofrimento no } \\
\text { trabalho: estudo qualitativo }\end{array}$ & $\begin{array}{c}\text { ACS } \\
144\end{array}$ & Brasil & $\begin{array}{l}\text { Identificar as situações geradoras de } \\
\text { prazer e de sofrimento no trabalho dos } \\
\text { Agentes Comunitários de Saúde. }\end{array}$ \\
\hline
\end{tabular}




\begin{tabular}{|c|c|c|c|c|c|}
\hline A14 & Guida et al., 2012 & \begin{tabular}{|l|} 
As relações entre saúde e \\
trabalho dos agentes de \\
combate às endemias da \\
Funasa: a perspectiva dos \\
trabalhadores
\end{tabular} & $\begin{array}{c}\text { ACE } \\
10\end{array}$ & Brasil & $\begin{array}{l}\text { Compreender a história da luta dos ACE } \\
\text { por reconhecimento social e justiça, bem } \\
\text { como conhecer os efeitos adversos na } \\
\text { saúde em razão das condições da } \\
\text { atividade de combate a endemias. }\end{array}$ \\
\hline A15 & $\begin{array}{c}\text { Barbosa; } \\
\text { Assunção; Araújo, } \\
2012 .\end{array}$ & \begin{tabular}{|l|} 
Distúrbios \\
musculoesqueléticos em \\
trabalhadores do setor \\
saúde de Belo Horizonte, \\
Minas Gerais, Brasil \\
\end{tabular} & $\begin{array}{l}\text { Trabalhadores da } \\
\text { Saúde, incluindo } \\
\text { ACS e ACE } \\
1808\end{array}$ & Brasil & $\begin{array}{l}\text { Avaliar a associação entre a prevalência } \\
\text { de distúrbios musculoesqueléticos e } \\
\text { características individuais, do emprego e } \\
\text { do trabalho em trabalhadores da rede } \\
\text { municipal de saúde. }\end{array}$ \\
\hline A16 & Dilélio et al., 2012. & \begin{tabular}{|l} 
Prevalência de transtornos \\
psiquiátricos menores em \\
trabalhadores da atenção \\
primária à saúde das \\
regiões Sul e Nordeste do \\
Brasil
\end{tabular} & $\begin{array}{l}\text { ACS } \\
4749\end{array}$ & Brasil & $\begin{array}{l}\text { Avaliar a prevalência de transtornos } \\
\text { psiquiátricos menores e fatores } \\
\text { associados entre trabalhadores da atenção } \\
\text { primária à saúde, nas regiões Sul e } \\
\text { Nordeste do Brasil. }\end{array}$ \\
\hline A17 & $\begin{array}{l}\text { Mascarenhas; } \\
\text { Prado; Fernandes, } \\
2013 .\end{array}$ & $\begin{array}{llr}\text { Fatores } & \text { associados } & \text { à } \\
\text { qualidade de vida } & \text { de } \\
\text { Agentes } & \text { Comunitários } & \text { de } \\
\text { Saúde } & & \end{array}$ & $\begin{array}{c}\text { ACS } \\
316\end{array}$ & Brasil & $\begin{array}{l}\text { Analisar a associação dos fatores } \\
\text { sociodemográficos, ocupacionais, } \\
\text { comportamentos de risco e saúde e da } \\
\text { qualidade de vida dos Agentes } \\
\text { Comunitários de Saúde do município de } \\
\text { Jequié, Bahia. }\end{array}$ \\
\hline A18 & Haikal et al., 2013 & $\begin{array}{|lrr|}\text { Qualidade } & \text { de } & \text { vida, } \\
\text { satisfação } & & \text { e } \\
\text { esforço/recompensa } & \text { no } \\
\text { trabalho, } & \text { transtornos } \\
\text { psíquicos } & \text { e níveis } & \text { de } \\
\text { atividade } & \text { física } & \text { entre } \\
\text { trabalhadores da } & \text { Atenção } \\
\text { Primária à Saúde } & & \\
\end{array}$ & $\begin{array}{c}\text { ACS } \\
752\end{array}$ & Brasil & $\begin{array}{l}\text { Investigar a qualidade de vida, a } \\
\text { satisfação e e o equilíbrio } \\
\text { esforço/recompensa no trabalho, presença } \\
\text { de transtornos psíquicos leves e níveis de } \\
\text { atividade física entre trabalhadores da } \\
\text { atenção primária à saúde. }\end{array}$ \\
\hline A19 & $\begin{array}{c}\text { Cipriano et al., } \\
2013\end{array}$ & $\begin{array}{l}\text { Relação entre distúrbio de } \\
\text { voz e trabalho em um grupo } \\
\text { de Agentes Comunitários } \\
\text { de Saúde }\end{array}$ & $\begin{array}{c}\text { ACS } \\
65\end{array}$ & Brasil & $\begin{array}{l}\text { Analisar a relação entre distúrbio de voze e } \\
\text { trabalho em um grupo de Agentes } \\
\text { Comunitários de Saúde (ACS). }\end{array}$ \\
\hline A20 & $\begin{array}{l}\text { Santos; Vargas; } \\
\text { Reis, } 2014 .\end{array}$ & $\begin{array}{|llr|}\begin{array}{l}\text { Estressores laborais em } \\
\text { agentes comunitários } \\
\text { saúde }\end{array} & \\
\end{array}$ & $\begin{array}{c}\text { ACS } \\
236\end{array}$ & Brasil & $\begin{array}{l}\text { Verificar a ocorrência do estresse laboral } \\
\text { em ACS da cidade de Aracaju (SE) }\end{array}$ \\
\hline A21 & $\begin{array}{c}\text { Darboe; Lin, Kuo, } \\
2016\end{array}$ & $\begin{array}{|ll|}\text { Effort-rewardim } & \\
\text { balanceand } & \text { self-rated } \\
\text { health among } & \text { Gambian } \\
\text { health care professionals. }\end{array}$ & $\begin{array}{c}\text { Oficiais de agentes } \\
\text { ambientais } \\
287\end{array}$ & Gâmbia & $\begin{array}{l}\text { Investigar a associação entre o estresse de } \\
\text { trabalho psicossocial percebido e auto } \\
\text { avaliação da saúde entre os profissionais } \\
\text { de saúde na Gâmbia }\end{array}$ \\
\hline A22 & Souza et al., 2016 & $\begin{array}{l}\text { Câncer de pele: hábitos de } \\
\text { exposição solar e alterações } \\
\text { cutâneas entre agentes de } \\
\text { saúde em um Município de } \\
\text { Minas Gerais. }\end{array}$ & $\begin{array}{c}\text { ACS e ACE } \\
56\end{array}$ & Brasil & $\begin{array}{l}\text { Identificar os fatores de risco, hábitos de } \\
\text { exposição solar, fotoproteção e as } \\
\text { alterações cutâneas em ACS e ACE no } \\
\text { município de Mateus Leme, Minas } \\
\text { Gerais, Brasil. }\end{array}$ \\
\hline A23 & $\begin{array}{l}\text { Almeida; Baptista; } \\
\text { Silva, } 2016 .\end{array}$ & $\begin{array}{|lllr|}\text { Cargas } & \text { de } & \text { trabalho } & \text { e } \\
\text { processo } & \text { de } & \text { desgaste } & \text { em } \\
\text { Agentes } & \text { Comunitários } & \text { de } \\
\text { Saúde } & & & \\
\end{array}$ & $\begin{array}{c}\text { ACS } \\
137\end{array}$ & Brasil & $\begin{array}{l}\text { Identificar as cargas de trabalho presentes } \\
\text { na atividade laboral do ACS e os } \\
\text { processos de desgaste decorrentes. }\end{array}$ \\
\hline A24 & Araújo et al., 2016 & \begin{tabular}{|lr|} 
Avaliação & \multicolumn{2}{c|}{ dermatológica } \\
de ACS & sujeitos à \\
fotoexposição em região \\
tropical do Brasil
\end{tabular} & $\begin{array}{c}\text { ACS } \\
83\end{array}$ & Brasil & $\begin{array}{l}\text { Investigar lesões de pele relacionadas ou } \\
\text { agravadas com a exposição solar em ACS } \\
\text { de dois municípios da região } \\
\text { metropolitana de Belém, Pará. }\end{array}$ \\
\hline A25 & $\begin{array}{c}\text { Leonelli et al., } \\
2017 .\end{array}$ & $\begin{array}{l}\text { Estresse percebido em } \\
\text { profissionais da Estratégia } \\
\text { Saúde da Família }\end{array}$ & $\begin{array}{c}\text { ACS } \\
450\end{array}$ & Brasil & $\begin{array}{l}\text { Avaliar o estresse percebido de } \\
\text { profissionais da Estratégia de Saúde da } \\
\text { Família e a associação com características } \\
\text { das equipes. }\end{array}$ \\
\hline A26 & Castro et al., 2017 & $\begin{array}{l}\text { Agentes Comunitários de } \\
\text { Saúde: perfil } \\
\text { sociodemográfico emprego } \\
\text { e satisfação com o trabalho } \\
\text { em um município do } \\
\text { semiárido baiano }\end{array}$ & $\begin{array}{c}\text { ACS } \\
145\end{array}$ & Brasil & $\begin{array}{l}\text { Conhecer o perfil sociodemográfico, } \\
\text { características do emprego e satisfação } \\
\text { com o trabalho de ACS do município de } \\
\text { Juazeiro, na Bahia. }\end{array}$ \\
\hline
\end{tabular}

Fonte: Elaborado pelos autores. 
Os resultados do estudo foram divididos em duas grandes categorias. A primeira abordou as condições de trabalho dos AS, e a segunda o adoecer destes trabalhadores no contexto laboral.

\section{Condicões de trabalho dos agentes de saúde}

As principais evidências sobre a saúde dos AS nos estudos destacam as condições de trabalho indesejadas que podem comprometer a saúde desses trabalhadores, bem como interferir na qualidade dos serviços prestados. Ficaram evidentes as questões referentes ao tempo dedicado ao trabalho, riscos ergonômicos, aspectos psicossociais e exposição a diferentes condições climáticas e a violência decorrente do trabalho realizado no território.

O tempo dedicado ao trabalho é um fator que interfere na qualidade de vida e saúde dos AS, pois se revela sempre superior ao tempo previsto nos contratos de trabalho, produzindo sobrecarga laboral (Castro et al., 2017).

Os ACS do município de Juazeiro, na Bahia, destacaram que morar e trabalhar na mesma comunidade, interfere no tempo de trabalho, comprometendo-o e tornando-o ainda mais demandante do que o previsto (Castro et al., 2017). Estudo realizado no Rio de Janeiro apontou que, mesmo fora do horário de trabalho, os ACS recebem solicitações constantes por parte da comunidade, seja em momentos de lazer, finais de semana, à noite e até mesmo nas férias (Nascimento; David, 2008). Tal condição por vezes acabam gerando constrangimento e tensões (Trindade et al., 2007).

Outro aspecto destacado nos estudos referiu-se a rua ser o ambiente de trabalho dos AS, caracterizado pelo espaço urbano que expõe esses trabalhadores à condições de trabalho inadequadas, marcadas, principalmente pelo deslocamento em calçadas estreitas e sem proteção, locais de difícil acesso, úmidos, escorregadios, sem pavimentação e ausência de saneamento básico (Trindade et al, 2007; Nascimento \& David,2008).

Queixas semelhantes também foram relatadas pelos trabalhadores comunitários de saúde do Canadá, com queixas em relação à infraestrutura precária das ruas e o trabalho ao ar livre, culminando em acidentes de trabalho e consequente absenteísmo. O risco de sofrer queda no Canadá foi significativo, afetando tanto o trabalhador quanto o serviço prestado (Drebit et al., 2010).

No contexto brasileiro, o trabalho do AS na rua também favorece aos acidentes de trabalho, como mostra estudo realizado em São Paulo, no qual 53\% dos ACS sofreram algum tipo de acidente laboral, sendo os mais frequentes aqueles com bicicleta, mordeduras de animais e quedas. Os acidentes típicos ${ }^{2}$ predominaram com $80 \%$ dos casos, $16,3 \%$ foram acidentes de trajeto $^{3}$ e 3,8\% decorrentes de doenças do trabalho ${ }^{4}$. Dentre os acidentes típicos e de trajeto, 76,6\% ocorreram nas vias públicas (Almeida et al., 2016).

Como destacado nos artigos, as características de trabalho dos ACS envolviam exposição a acidentes durante o deslocamento, principalmente a pé ou com bicicletas. As longas caminhadas e o peso dos materiais carregados que são necessários para o desenvolvimento do trabalho foram apontados como riscos ergonômicos presentes na atividade laboral dos AS (Trindade et al, 2007; Nascimento; David, 2008; Barbosa, Assunção; Araújo, 2012; Mascarenhas et al., 2013; Almeida et al., 2016).

Quanto as condições ambientais e climáticas também podem repercutir na saúde dos AS. Os ACS são expostos à poeira das ruas, fumaça dos veículos, de fábricas e de queimadas de lixo (Trindade et al., 2007; Souza et al., 2016). Em estudo realizado em São Paulo foi encontrada associação entre a exposição à poeira das ruas e queixas vocais. Alterações vocais como rouquidão, falta de ar, falha na voz, voz grossa, voz fina, garganta seca e cansaço ao falar também foram destacadas em estudo com ACS,

\footnotetext{
2. É o que ocorre na execução do trabalho (Art. 19 da Lei 8213/91).

3. É o que ocorre no percurso da residência para o trabalho ou vice-versa (Art. 19 da Lei 8213/91).

4. É adquirida ou desencadeada em função de condições especiais em que o trabalho é realizado e com ele se relacione diretamente. (Art. 20 da Lei 8213/91).
} 
representando $42,9 \%$ das queixas entre essa categoria de profissionais. Essas queixas estavam relacionadas a fatores ambientais e organizacionais do trabalho (Cipriano et al., 2013).

A exposição frequente a radiação solar durante a atividade laboral foi um aspecto destacado com ênfase nos artigos, sendo apontado como algo que coloca em risco a saúde dos AS. Em geral, eles se expõem aos raios solares por mais de cinco horas por dia e em horários onde esses raios são mais danosos à saúde, o que favorece queimaduras solares, envelhecimento precoce, rugas, sardas e em longo prazo, predispõe ao câncer de pele (Trindade et al., 2007; Nascimento; David., 2008; Lima et al., 2010; Almeida et al., 2016; Araújo et al., 2016; Souza et al., 2016).

Assim, as longas caminhadas sob o sol, bem como os dias frios e chuvosos podem tornar o trabalho árduo e fatigante, merecendo atenção. As condições climáticas presentes no ambiente laboral do AS não podem ser controladas, mas é possível criar medidas protetoras para o seu adequado enfrentamento (Lima et al., 2010; Araújo et al., 2016; Souza et al., 2016). No entanto, apesar dessa exposição ser evidenciada consistentemente e os métodos de proteção também estarem disponíveis (como os filtros solares, bonés, chapéus, sombrinhas e roupas fotoprotetoras), os estudos apontam que, em geral, a gestão do trabalho não tem assegurado essa proteção, ficando os trabalhadores expostos ou tendo eles próprios que prover recursos financeiros para adoção dessa medida (Lima et al., 2010; Araújo et al., 2016). Portanto, este é um tema que precisa ser incorporado à discussão sobre o trabalho dos AS.

Visitas domiciliares constituem elemento central da atividade do AS, proporcionando sentimento de responsabilidade pelas famílias. No entanto, nem sempre essa atividade pode ser cumprida como prevista, em função de condições de trabalho desfavoráveis. Sobre este aspecto, estudo com ACS do Rio de Janeiro- RJ apontou condições que dificultavam o trabalho e colocava esses profissionais em situação de vulnerabilidade de saúde durante o atendimento em domicílio em função do descaso pelos moradores, dificuldade para beber água e contato direto com pessoas doentes (Nascimento \& David, 2008). Em outro estudo realizado no Rio Grande do Sul, os ACS relataram sentir-se hostilizados e desvalorizados, além de deparar-se, nos domicílios, com higiene precária, barulho excessivo, presença de animais e fumaça (Trindade et al., 2007).

Outro aspecto relativo às visitas domiciliares referiu-se à inconstância dos horários que dependem da disponibilidade das famílias (Wai \& Carvalho, 2009; Cipriano \& Ferreira, 2011). Assim, a rotina dos ACS torna-se comprometida e imprevisível, em função disso, desencadeiam-se sintomas de estresse, insatisfação, além de não permitir o estabelecimento de rotinas de descanso, alimentação e convívio familiar (Castro et al., 2017), o que acarreta impactos sobre a qualidade de vida desses trabalhadores.

A organização do trabalho também foi citada em como fator que gera sobrecarga física e psíquica, como a falta de tempo para todas as atividades, ritmo intenso, exigência por produtividade, excesso de visitas domiciliares, incapacidade de responder às demandas e dificuldade de relação interpessoal com a equipe e famílias (Wai; Carvalho, 2009; Cipriano et al., 2013; Mascarenhas et al., 2013; Santos et al., 2014; Almeida et al., 2016).

Os estudos desta revisão apontam a violência como um problema vivenciado pelos AS que residem no mesmo território onde atuam (Nascimento \& David, 2008; Lancman et al., 2009; Guida et al., 2012). Enfrentam um contexto de violência social suscitada pelo tráfico de drogas, miséria e desigualdade social. Convivem com o medo, pressão e incertezas que, por sua vez, suscitam desgaste entre esses profissionais (Guida et al., 2012). Desta forma, o trabalho torna-se ameaçado e limitado diante da violência, pelo medo da exposição aos agressores (Lopes et al., 2012).

Além desse tipo de violência mais ampla decorrente de processos sociais mais gerais, os AS vivenciavam situações de violência intradomiciliar. Frequentemente, os AS presenciam brigas e agressões dentro das famílias, causando desconforto e sentimento de impotência (Cipriano et al., 2013). São alvos da violência verbal, na forma de agressões verbais, de hostilidade, desprezo e desvalorização, quando não atendem às expectativas da comunidade, chegando em alguns momentos a sofrerem violência física nos domicílios (Trindade et al., 2007; Wai \& Carvalho, 2009; Santos \& David, 2011; Cipriano et al., 2013). 


\section{O adoecer dos Agentes de Saúde}

Com relação à situação de saúde dos AS, o adoecimento mental foi predominantemente abordada. Entre os estudos revisados, dez trataram dos transtornos mentais relacionados ao trabalho, enquanto três investigaram problemas de pele, devido à exposição solar, dois, as queixas vocais, um, os distúrbios músculos esqueléticos relacionados ao trabalho e um, os acidentes de trabalho, em decorrência de quedas.

O sofrimento mental gerado pelo trabalho foi relatado pelos AS do Rio Grande do Sul, com destaque para os afastamentos por depressão (Lopes et al., 2012). Entre os AS do Rio de Janeiro foram relatados sintomas de estresse, ansiedade e tensões (Nascimento \& David, 2008). No cenário internacional, entre os trabalhadores de saúde comunitária da Gambia, identificou-se alta prevalência de estresse psicossocial, devido às condições de trabalho (Darboe et al., 2016).

Estudo realizado no Município de Aracaju, SE, verificou-se que 61,4\% dos AS apresentavam alguma manifestação de estresse. Em relação aos sintomas mais crônicos, predominaram os de natureza psicológica: insônia (10,9\%), sensação de cansaço excessivo (9,4\%); e irritabilidade (7,2\%) (Santos; Vargas; Reis, 2014). A síndrome de burnout, caracterizada por estresse crônico, desinteresse pelo trabalho e autoestima comprometida foi observada entre os AS em diferentes estudos (Telles \& Pimenta, 2009; Silva \& Menezes, 2008; Malakout et al., 2011). Assim como prevalências de Transtornos Mentais Comuns (TMC) também foram identificadas entre os AS (Silva; Menezes, 2008; Dilélio et al., 2012).

Outro estudo realizado em 12 Unidades Básicas de Saúde da cidade de São Paulo mostrou que os ACS estão entre os trabalhadores da equipe que apresentam elevados níveis de estresse, que estão associados ao tempo de trabalho igual ou superior a um ano na mesma equipe, ser do sexo feminino, não ser praticante de nenhuma religião e atuarem em equipes de saúde incompletas (Leonelli et al., 2017).

A insatisfação no trabalho foi observada como fator que favorece o adoecimento dos ACS, como evidenciou o estudo realizado no município de Montes Claros, MG (Haikal et al., 2013). Fatores como sobrecarga no trabalho e dificuldade de gerenciar o tempo foram citados como causadores da insatisfação, estando associados a sintomas de ansiedade, insônia, fadiga e sofrimento psicoemocional (Cipriano et al., 2013). No cenário internacional, diferentemente do encontrado nos estudos brasileiros, os AS da China, por exemplo, apresentaram nível elevado de satisfação no trabalho. Os fatores relacionados à maior satisfação foram autonomia no trabalho, poder decisório, bom relacionamento interpessoal com os colegas de trabalho, apoio da gestão e suporte social. Além destes, a remuneração financeira e o ajuste da carga horária de trabalho também estiveram associadas ao maior grau de satisfação com a atividade laboral exercida (Ge et al., 2011).

Os AS também estavam suscetíveis às doenças osteomusculares, provenientes das longas caminhadas a pé ou de bicicleta, carregamento de peso e posturas inadequadas que levam às queixas de dores em membros inferiores, dor na coluna, desvios da coluna cervical, dores musculares, varizes e cefaléias (Nascimento \& David, 2008; Trindade et al., 2007; Cipriano; Ferreira, 2011; Mascarenhas et al., 2013).

\section{Discussão}

Neste estudo evidenciou-se que as condições de trabalho e de saúde dos AS apresentaram diferenciais entre países. Com base nos artigos revisados, o trabalho do AS no Brasil é marcado por condições de trabalho inadequadas as quais expõem esses trabalhadores e trabalhadoras a fatores de riscos biológico, físicos, químicos, ergonômicos, acidentes, doenças relacionadas ao trabalho e violência. Os estudos apontaram adoecimento por Lesões por Esforços Repetitivos (LER), Distúrbios Osteomusculares Relacionados ao Trabalho (DORT), problemas de pele, acidentes durante o desenvolvimento da atividade laboral e transtornos mentais relacionados ao trabalho.

O trabalho dos AS exige atenção, agilidade e esforço para superar o cansaço físico e mental de modo a manter o bom desempenho profissional, no cuidado prestado aos usuários dos serviços. Os dados revelam o interesse e preocupação dos 
pesquisadores brasileiros em realizar estudos sobre a saúde desta categoria, em função da importância destes profissionais para a saúde pública, por um lado, e da precariedade das condições de trabalho e da ausência de medidas de proteção à saúde, por outro (Alcântra \& Assunção, 2016).

Observa-se nesses profissionais sentimentos de desvalorização diante dos demais membros da equipe de saúde, sobrecarga de trabalho, por morar e trabalhar na mesma comunidade, ausência de programas de formação continuada, capacitação e supervisão, fatores estes contributivos ao surgimento de sentimentos de auto-depreciação e sofrimento psíquico (Cremonese et al., 2013).

$\mathrm{O}$ ambiente laboral dos AS associado às condições de trabalho constituem fatores estressores que podem repercutir negativamente na saúde mental e psicoemocional desses profissionais. A exposição frequente a condições de estresse pode levar a ocorrência da síndrome de burnout e ao surgimento dos TMC e depressão (Mota et al., 2014; Alcântara \& Assunção, 2016). Esses agravos à saúde têm sido associados à alta demanda psicológica, agressões contra o trabalhador e dificuldades nas relações interpessoais (Araújo et al., 2016).

Por trabalharem na mesma comunidade em que residem, os AS enfrentam a dualidade da vida privada e pública que se sobrepõe, e que, perdendo seus limites, restringem os espaços de privacidade dos AS. As constantes solicitações por parte da comunidade fora do horário de trabalho do AS geram desgaste no profissional e tem sido considerada uma condição de trabalho indesejada. A ausência de privacidade é fator que leva esses profissionais, em muitos momentos, a mudarem sua rotina e estilo de vida por causa da comunidade (Menegussi et al., 2014). Desta forma, observa-se que este é um trabalho que jamais cessa, pois os AS convivem em um ambiente de trabalho desprovido de limites entre o ambiente de trabalho e a vida pessoal.

Nesta fronteira entre cuidar do outro e o cuidar de si são gerados conflitos, que expressam-se através do sofrimento emocional, insatisfação e desmotivação pelo trabalho (Ursine et al., 2010). Quanto mais frequente são as demandas fora do tempo de trabalho profissional, mais o trabalho se torna fonte de sofrimento psíquico, podendo se tornar patogênico.

É importante ressaltar que o adoecimento do AS pode ter impactos não apenas individuais e familiares, mas pode afetar a saúde das comunidades assistidas em função das limitações impostas pelo próprio adoecimento, pelo absenteísmo ao trabalho ou, até mesmo, pelo abandono da profissão (Jardim \& Lacman, 2009; Tinoco, 2015).

Embora a proposta da ESF tenha estabelecido essa exigência com a finalidade de aproximar a comunidade do serviço, vivenciar a realidade com todas as suas particularidades, favorecendo o papel do AS como articulador dos serviços de saúde, tal estratégia, por outro lado, acabou por impactando no tempo fora do trabalho, o que gerou sobrecarga de trabalho para estes profissionais. As visitas domiciliares, enquanto atribuição primordial dos AS demandam muitas horas de trabalho. Assim, outras atividades ficam comprometidas, o que leva esses profissionais a trabalhar fora do horário de trabalho para alcançar metas préestabelecidas (Tinoco, 2015).

Quanto ao trabalho exposto a raios solares, muitos AS se expõem em horários críticos e por longas horas a radiação solar. A exposição de forma indevida aos raios solares predispõe às lesões de pele. Estima-se que $80 \%$ dos melanomas em AS são provenientes da exposição aos raios solares (Brasil, 2006). Estudo conduzido em Belém no Pará apontou que os ACS têm uma exposição solar excessiva ( 2 a 6 horas semanais). Mais de um terço dos AS se expõem por mais de 06 horas, com horário de maior exposição a radiação solar entre 10 e 15 horas (Malcher et al., 2019).

Nesta revisão, ficaram evidentes as situações de violência vivenciada pelos AS, observada principalmente em estudos de natureza qualitativa. A violência no trabalho entre os trabalhadores da atenção primária de Belgrado, na Sérvia, foi também elevada atingindo 52,6\% dos trabalhadores estudados. $\mathrm{O}$ abuso verbal foi o mais prevalente (43,5\%), seguido da violência física (18,3\%) (Fisekovic et al., 2015). Assim como na Al-Hassa, Arábia Saudita, 27,7\% dos trabalhadores/as da atenção primária já sofreu algum tipo de violência: $92,1 \%$ relataram violência emocional, sendo 54,2\% especialmente abuso verbal. Os fatores contributivos para esse quadro foram a insatisfação com a demanda do serviço e falta de penalidade dos agressores. Situações 
de violência, gerando insatisfação no trabalho e desmotivação (El-gilany et al., 2010), corroborando com os achados desta revisão integrativa que revelam a violência verbal por insatisfação da comunidade com os serviços de saúde prestados pela unidade de saúde.

No Brasil, a violência urbana e suas representações sociais, além do impacto sobre a saúde do trabalhador, interferem na produção do cuidado em saúde, na conformação do trabalho e de vínculos, impondo limites ao exercício profissional dos AS (Almeida et al., 2019). Estudos apontam que os AS não se sentem suficientemente preparados para atendimento a demandas referente ao consumo de drogas e uso abusivo álcool e a violência intrafamiliar no território, embora sejam questões recorrentes em seu cotidiano (Almeida et al., 2019; Garbin et al., 2014).

\section{Considerações Finais}

Este estudo possibilitou evidenciar entre os AS os fatores que determinam o processo de saúde /doença e sua relação com as condições de trabalho através da síntese de estudos científicos produzidos, ampliando a compreensão acerca do fenômeno investigado.

A aproximação com a comunidade e o território expõe os AS à violência e falta de infraestrutura dos territórios que impactam negativamente no processo de trabalho destes indivíduos, além das próprias condições de trabalho, como as relações interpessoais, sobrecarga de trabalho, falta de valorização e não reconhecimento do trabalho pela comunidade. Os principais resultados desta revisão integrativa revelaram que os aspectos psicossociais estão diretamente envolvidos no processo de desgaste e adoecimento dos AS.

Nesta direção, em relação ao adoecimento pelo trabalho, a maioria dos estudos abordou os transtornos mentais relacionados ao trabalho, como síndrome de burnout, TMC, estresse percebido e sofrimento psíquico, relacionado ao seu contexto laboral. Aspectos importantes para serem investigados são as queixas vocais relacionadas ao trabalho, os acidentes durante o trabalho e problemas de pele apontados nesta revisão, sendo necessários mais estudos para aprofundar o conhecimento acerca destes aspectos, além da implantação de um sistema de informação para registro dos casos.

Os resultados apontados evidenciam a necessidade de se intervir nos processos e na organização do trabalho, a fim de promover ambientes saudáveis e, desta forma, contribuir para a qualidade de vida dos AS. Identificou-se a necessidade de implantar programas específicos voltados para a proteção e promoção da saúde dos AS de forma continuada, e com a inserção deles no processo e nas ações de saúde do trabalhador.

Este estudo evidenciou ainda uma importante lacuna científica no que se refere a ausência de estudos de intervenção e observacionais prospectivos. Assim, ressalta-se a necessidade de estudos com esses delineamentos com a finalidade de conhecer melhor os efeitos da exposição ocupacional na saúde ao longo da trajetória profissional e testar estratégias de intervenção que possam melhorar as condições laborais e reestruturar a organização do trabalho dos AS, bem como melhorar a saúde e a qualidade de vida desses trabalhadores.

\section{Referências}

Alcântara, M. A. \& Assunção, A. Á. (2016). Influência da organização do trabalho sobre a prevalência de transtornos mentais comuns dos agentes comunitários de saúde de Belo Horizonte. Revista Brasileira de Saúde Ocupacional ,41 (21)e2.

Almeida, J. F., Peres, M. F. T. \& Fonseca, T. L. (2019). O território e as implicações da violência urbana no processo de trabalho dos agentes comunitários de saúde em uma unidade básica. Saúde e Sociedade, 28 (1).

Almeida, S., Baptista, P. \& Silva. A. (2016). Cargas de trabalho e processo de deformação em Agentes Comunitários de Saúde. Revista Escola de Enfermagem da USP, São Paulo, 50(1)95-103.

Araújo, F. C., Sousa, B. R. de M., Leite, G. G., Freitas, L. C. de, Lemos, E. L. da C. \& Pires, C. A. A. (2016). Avaliação dermatológica de agentes comunitários de saúde sujeitos à fotoexposição em região tropical do Brasil. Scientia Medica, 26(4). 
Barbosa, R. E. C., Assunção, A. Á. \& Araújo, T. M. (2012). Distúrbios musculoesqueléticos em trabalhadores do setor saúde de Belo Horizonte, Minas Gerais, Brasil. Cadernos de Saúde Pública, Rio de Janeiro, 28 (8)1569-1580.

Bardin, L. (2009). Análise de Conteúdo. Edições 70. LTDA

Brasil. Ministério da Saúde. Secretaria de Atenção à Saúde. Instituto Nacional de Câncer. Coordenação de Prevenção e Vigilância. (2006). A situação do câncer no Brasil, Rio de Janeiro, Instituto Nacional de Câncer - INCA Coordenação de Prevenção e Vigilância - Conprev.

Botelho, L. L. R., Cunha, C. C. de A. \& Macedo, M. (2011). O método da revisão integrativa nos estudos organizacionais. Gestão E Sociedade, 5(11), 121-136.

Castro, T. A., Davoglio, R. S., Nascimento, A. A. J., Santos, K. J. S., Coelho, G. M. P. \& Lima, K. S. B. (2017). Agentes Comunitários de Saúde: perfil sociodemográfico, emprego e satisfação com o trabalho em um município do semiárido baiano. Cadernos e Saúde Coletiva, 25(3), 294-301.

Cipriano, F. G., Ferreira, L. P., Servilha, E. A. M. \& Marsiglia, R. M. G. (2013). Relação entre distúrbio de voz e trabalho em um grupo de Agentes Comunitários de Saúde. CoDAS, 25(6)548-56.

Cipriano, F. G. \& Ferreira, L. P. (2011). Queixas de voz em agentes comunitários de saúde: correlação entre problemas gerais de saúde, hábitos de vida e aspectos vocais. Revista Sociedade Brasileira de Fonoaudiologia, 16(2)132-9.

Cremonese, G. R., Motta, R. F. \& Traesel, E. S. (2013). Implicações do trabalho na saúde mental dos Agentes Comunitários de Saúde. Cadernos De Psicologia Social Do Trabalho, 16(2), 279-293.

Darboe, A., Lin, IF. \& Kuo, HW. (2026). Effort-rewardimbalanceand self ratedhealthamongGambianhealthcareprofessionals. BMC Health Serv Res, 16(125).

Dilélio, A. S., Facchini, L. A., Tomasi, E., Silva, S. M., Thumé, E., Piccini, R. X., Silveira, D. S., Maia, M. F. S., Osório, A., Siqueira, F. V., Jaardim, V. M. R., Lemões, M. A. M. \&Borges, C. L. S. (2012). Prevalência de transtornos psiquiátricos menores em trabalhadores da atenção primária à saúde das regiões Sul e Nordeste do Brasil. Caderno de Saúde Pública, 28(3) 503-514.

Drebit, S., Shajari, S., Alamgir, H., Yu, S. \&Keen, D. (2010). Occupationalandenvironmentalriskfactors for fallsamongworkers in thehealthcare sector. Ergonomics, 53(4), 525-536.

El-Gilany, A.-H., El-Wehady, A., \&Amr, M. (2010). ViolenceAgainstPrimary Health CareWorkers in Al-Hassa, Saudi Arabia. JournalofInterpersonalViolence, 25(4), 716-734.

Facchini, L. A. (1993). Uma contribuição da epidemiologia: o modelo de determinação social aplicado à saúde do trabalhador. In: Buschinelli, J. T. P., Rocha, L. E. \& Rigotto, R. M. (orgs.). Isto é trabalho de gente? Vida, doença e trabalho no Brasil. VOZES,178-186.

Fisekovic, M. B., Trajkovic, G. Z., Bjegovic-Mikanovic, V. M. \& Terzic-Supic, Z. J. (2015). Does workplaceviolenceexist in primaryhealthcare? EvidencefromSerbia. Europeanjournalofpublichealth, 25(4), 693-698.

Ge, C., Fu, J., Chang, Y. \& Wang, L. (2011). FactorsassociatedwithjobsatisfactionamongChinesecommunityhealthworkers: a cross-sectionalstudy. BMC publichealth, 11,884 .

Guida, H. F. S., Souza, K. R., Santos, M. B. M., Silva, S. M. C. L., \& Silva, V. P. (2012). As relações entre saúde e trabalho dos agentes de combate às endemias da Funasa: a perspectiva dos trabalhadores. Saúde e Sociedade, 21(4)858-870.

Haikal D.S.'A., Santa-Rosa, T. T. A., Oliveira, P. E. A., Sales, L. O. S.,Silveira, L. O., Pereira, A. C. A., Macedo. C. C. S., Ferreira, R. C., Martins, A. M. E. B. L. \&Ferreira, E. F. (2013). Qualidade de vida, satisfação e esforço/recompensa no trabalho, transtornos psíquicos e níveis de atividade física entre trabalhadores da Atenção Primária à Saúde. RevistaAtençãoPrimária à Saúde,16(3)301-312.

Jardim, T. A., Lancman, S. (2009). Subjective aspects of living and working within the same community: the realities experienced by community healthcare agents. Interface:Comunicação, Saúde, Educação,13(28)123-35.

Lancman, S., Ghirardi, M. I. G., Castro, E. D., \&Tuacek, T. A. (2009). Repercussões da violência na saúde mental de trabalhadores do Programa Saúde da Família. Revista de Saúde Pública, 43(4) 682-8.

Leonelli, L. B., Andreoni, S., Martins, P., Kozasa, E. H., Salvo, V. L., Sopezki, D., Montero-Marin, J., Garcia-Campayo, J. \& Demarzo, M. M. P. (2017). Estresse percebido em profissionais da Estratégia Saúde da Família. Revista Brasileira de Epidemiologia, 20(2) 286-298.

Lima, A. G., Silva, A. M. M. da, Soares, C. E. C., Souza, R. A. X. \& Souza, M. C. M. R. (2010). Fotoexposição solar e fotoproteção de agentes de saúde em município de Minas Gerais. Revista Eletrônica De Enfermagem, 12(3), 478-82.

Lopes, D. M. Q., Beck, D. M. Q., Colomé, C. L., Prestes, F. C., Weiller, T. H., Colomé, J. S. \& Silva, G. M. (2012). Agentes Comunitários de Saúde e as vivências de prazer - sofrimento no trabalho: estudo qualitativo. Revista da Escola de Enfermagem da USP, 46, (3), 633-40.

Malakouti, S. K., Nojomi, M., Salehi, M., \&Bijari, B. (2011). Job stress andburnoutsyndrome in a sampleof rural healthworkers, behvarzes, in tehran, iran. Iranianjournalofpsychiatry, 6(2), 70-74.

Malcher, C. M. S. R., Tembra, A. L., Amorim, F. C., Souza, T. R. M. de, \& Pessoa, M. dos S. (2019). Fotoproteção em Agentes Comunitários de Saúde (ACS) de Belém-PA. Revista Brasileira De Medicina De Família E Comunidade, 14(41), 1798.

Mascarenhas, C. H. M., Prado, F. O. \& Fernandes, M. H. (2013).Fatores associados à qualidade de vida de Agentes Comunitários de Saúde. Ciência \& Saúde Coletiva, 18(5)1375-1386.

Mendes, K. D. S. Silveira, R. C. C. P. \& Galvão, C. M. (2008). Revisão integrativa: método de pesquisa para a incorporação de evidências na saúde e na enfermagem. Texto e Contexto Enfermagem, 17(4)758-764. 
Menegussi, J. M., Ogata, M. N. \& Rosalini, M. H. P.(2014). O agente comunitário de saúde como morador, trabalhador e usuário em são Carlos, São Paulo. Revista Trabalho, Educação e Saúde, 12 (1) 87-106.

Mota, C. M., Dosea, G. S. \& Nunes, P. S.(2014). Avaliação da presença da Síndrome de Burnout em Agentes Comunitários de Saúde no município de Aracaju, Sergipe, Brasil. Ciência \& Saúde Coletiva, 19(12) 4719-4726.

Nascimento, G. M. \& David, H. M. S. L. (2008). Avaliação de riscos no trabalho dos agentes comunitários de Saúde: um processo participativo. Revista Enfermagem UERJ, v. 16(4) 550-6.

Roman, A. R. \& Friedander, M. R. (1998). Revisão integrativa de pesquisa aplicada à enfermagem. Cogitare Enfermagem, 3(2)109-112.

Samudio, J. L. P., Brant, L. C., Martins, A. C. F. D. C., Vieira, M. P. \& Sampaio, C. A. (2017). Agentes comunitários de saúde na atenção primária no Brasil: multiplicidade de atividades e fragilização da formação. Revista Trabalho. Educação e Saúde, 15(3)745-769.

Santos, L. F. B. \& David, H. M. S. L. (2011). Percepções do estresse no trabalho pelos agentes comunitários de saúde. Revista Enfermagem UERJ, 19(1) 52-7.

Santos, I. E. R., Vargas, M. M. \& Reis, F. P. (2014). Estressores laborais em agentes comunitários de saúde. Revista Psicologia Organizações e Trabalho, 14(3), 324-335.

Santos, M. G., Santos, M. G.,Ceretta, L. B., Schwalm, M. T.,Dagostim, V. S. \&Soratto, M. T (2015). Desafios enfrentados pelos agentes comunitários de saúde na estratégia saúde da família. Revista Inova Saúde, 4(1)26-46.

Silva, A. T. \& Menezes, P. R. (2008). Esgotamento profissional e transtornos mentais comuns em agentes comunitários de saúde. Revista de Saúde Pública, 42(5)921-9.

Souza, M. C. M. R., Hort, G. T., Melo, E. S. \& Rocha, F. D. B. (2016). Câncer de pele: hábitos de exposição solar e alterações cutâneas entre agentes de saúde em um Município de Minas Gerais. Revista de Enfermagem do Centro-oeste Mineiro, Minas Gerais, 1(6)1945-1956.

Telles, S. H. \& Pimenta, A. M. C. (2009). Síndrome de Burnout em agentes comunitários de saúde e estratégias de enfrentamento. Saúde e Sociedade, 18(3)467478.

Tinoco, M. M. (2025). A relação saúde/doença no processo de trabalho dos Agentes Comunitários de Saúde: uma revisão de literatura. Dissertação. Mestrado em Saúde Pública. Escola Nacional de Saúde Pública Sergio Arouca, Fundação Oswaldo Cruz, Rio de Janeiro.

Trindade, L. L., Gonzales, R. M. B., Beck, C. L. C. \&Lautert, L. (2007). Cargas de trabalho entre os agentes comunitários de saúde. Revista Gaúcha de Enfermagem, 28(4)473-9.

Ursine, B. L., Trelha C. S. \& Nunes E. F. P. A. (2010). O Agente Comunitário de Saúde na Estratégia de Saúde da Família: uma investigação das condições de trabalho e da qualidade de vida. Revista Brasileira de Saúde Ocupacional, 35(122)327-339.

Wai, M. F. P. \& Carvalho A. M. P. (2009). O trabalho do agente comunitário de saúde: fatores de sobrecarga e estratégias de enfrentamento. Revista Enfermagem UERJ, 17(4)563-8. 